\title{
A Comparative Study of Adomain Decompostion Method and He-Laplace Method
}

\author{
Badradeen A. A. Adam1,2 \\ ${ }^{1}$ Department of Mathematics, Faculty of Education, University of Khartoum, Omdurman, Sudan \\ ${ }^{2}$ Department of Mathematics, Northwest Normal University, Lanzhou, China \\ Email: bdr uofk@yahoo.com, badradeenabaker@uofk.edu
}

Received 8 October 2014; revised 29 October 2014; accepted 18 November 2014

Copyright (@ 2014 by author and Scientific Research Publishing Inc.

This work is licensed under the Creative Commons Attribution International License (CC BY). http://creativecommons.org/licenses/by/4.0/

(c) (1) Open Access

\begin{abstract}
In this paper, we present a comparative study between the He-Laplace and Adomain decomposition method. The study outlines the significant features of two methods. We use the two methods to solve the nonlinear Ordinary and Partial differential equations. Laplace transformation with the homotopy method is called He-Laplace method. A comparison is made among Adomain decomposition method and He-Laplace. It is shown that, in He-Laplace method, the nonlinear terms of differential equation can be easy handled by the use He's polynomials and provides better results.
\end{abstract}

\section{Keywords}

Adomain Decomposition Method, He-Laplace Transform Method, Homotopy Perturbation Method, Ordinary Differential Equation, Partial Differential Equations, He's Polynomials

\section{Introduction}

This paper outlines a reliable Comparison between two powerful methods that were recently developed. The first is Adomain decomposition method (ADM) developed by Adomain in [1] [2], and used heavily in the literature in [3]-[10] and the references therein. The second is He-Laplace method, an elegant combination of the Laplace transformation, the homotopy perturbation method and He's polynomials. The use of He's polynomial in nonlinear term was first introduced by Ghorbani [11]. The proposed algorithm provides the solution in a rapid convergent series which may lead to the solution in a closed form. The two methods give rapidly convergent series with specific significant features for each scheme. Some of the classical analytic methods are lyapunov's ar- 
tificial small parameter method [12] perturbation techniques [13] [14] and Hiroa bilinear method [15] [16]. In recent years, many authors have paid attention to study the solution of nonlinear partial differential equation by using various methods. Variational iteration method, He's semi inverse method [17] and the differential transform method, etc. are among these. The main objective is to introduce a comparative study to nonlinear ordinary differential and partial differential equations by using adomain decomposition method and He-Laplace method.

This paper contains basic idea of homotopy pertaturbation method and He-Laplace method in Section 2, Adomain decomposition method in 3, Application in 4 and conclusion and discussions in 5 respectively.

\section{Basic Idea of Homotopy Perturbation Method and He-Laplace Method}

\subsection{Homotopy Perturbation Method}

Consider the following nonlinear differential equation

$$
A(y)-f(r)=0, \quad r \in \Omega
$$

with boundary conditions of

$$
B\left(y, \frac{\partial y}{\partial n}\right)=0, \quad r \in \Gamma
$$

where $A, B, f(r)$ and $\Gamma$ are a general differential operator, a boundary operator, a known analytic function and the boundary of the domain $\Omega$, respectively.

The operator A can generally be divided into a linear part L and a nonlinear part M. Equation (1) may therefore be written as:

$$
L(y)+M(y)-f(r)=0
$$

By the homotopy technique, we construct a homotopy $v(r, p): \Omega \times[0,1] \rightarrow R$ which satisfies:

$$
H(v, p)=(1-p)\left[L(v)-L\left(y_{0}\right)\right]+p[A(v)-f(r)]=0
$$

or

$$
H(v, p)=L(v)-L\left(y_{0}\right)+p L\left(y_{0}\right)+p[M(v)-f(r)]=0
$$

where $p \in[0,1]$ is an embedding parameter, while $y_{0}$ is an initial approximation of Equation (1), which satisfies the boundary conditions. Obviously, from Equatons (4) and (5), we will have:

$$
\begin{aligned}
& H(v, 0)=L(v)-L\left(y_{0}\right)=0 \\
& H(v, 1)=L(v)-L\left(y_{0}\right)=0
\end{aligned}
$$

The changing process of $P$ from zero to unity is just that of $v(r, p)$ from $y_{0}$ to $y(r)$. In topology, this is called deformation, while $L(v)-L\left(y_{0}\right)$ and $A(v)-f(r)$ are called homotopy. If the embedding parameter pis considered as a small parameter, applying the classical perturbation technique, we can assume that the solution of Equations (4) and (5) can be written as a power series in $p$ :

$$
v=v_{0}+p v_{1}+p^{2} v_{2}+p^{3} v_{3}+\cdots+\infty
$$

Setting $p=1$ in Equations (8), we have

$$
y=\lim _{p \rightarrow 1} v=v_{0}+v_{1}+v_{2}+\cdots
$$

The combination of the perturbation method and the homotopy method is called the HPM, which eliminates the drawbacks of the traditional perturbation methods while keeping all its advantages. The series (9) is convergent for most cases. However, the convergent rate depends on the nonlinear operator $A(v)$. Moreover, He [18] made the following suggestions: 
1) The second derivative of $M(v)$ with respect to must be small because the parameter may be relatively large, i.e. $p \rightarrow 1$.

2) The norm of $L^{-1}\left(\frac{\partial M}{\partial v}\right)$ must be smaller than one so that the series converges.

\subsection{He-Laplace Method}

Consider the following nonlinear differential equation (IVP):

$$
\begin{gathered}
y^{\prime \prime}+p_{1} y^{\prime}+p_{2} y+p_{3} f(y)=f(x) \\
y(0)=\alpha, \quad y^{\prime}(0)=\beta
\end{gathered}
$$

where $p_{1}, p_{2}, p_{3}, \alpha, \beta$ are constant. $f(y)$ is a nonlinear function and $f(x)$ is the source term. Taking Laplace transformation (denoted throughout this paper by $L$ ) on both side of Equation (10), we have

$$
L\left[y^{\prime \prime}\right]+L\left[p_{1} y^{\prime}\right]+L\left[p_{2} y\right]+L\left[p_{3} f(y)\right]=L[f(x)]
$$

By using linearity of Laplace transformation, the result is

$$
L\left[y^{\prime \prime}\right]+L p_{1}\left[y^{\prime}\right]+L p_{2}[y]+L p_{3}[f(y)]=L[f(x)]
$$

Applying the formula on Laplace transform, we obtain

$$
s^{2} L[y]-s y(0)-y^{\prime}(0)+p_{1} L\{s L[y]-y(0)\}+p_{2} L[y]+p_{3} L[f(y)]=L[f(x)]
$$

Using initial conditions in Equation (14), we have

$$
\left(s^{2}+p_{1} s\right) L[y]=\alpha s+\beta+\alpha p_{1}-p_{2} L(y)-p_{3} L[f(y)]+L[f(x)]
$$

Or

$$
L[y]=\frac{\left(\alpha s+\beta+\alpha p_{1}\right)}{\left(s^{2}+p_{1} s\right)}-\frac{p_{2}}{\left(s^{2}+p_{1} s\right)} L[y]-\frac{p_{3}}{\left(s^{2}+p_{1} s\right)} L[f(y)]+L[f(x)]
$$

Taking inverse Laplace transform, we have

$$
y(x)=F(x)-L^{-1}\left(\frac{p_{3}}{s^{2}+p_{1} s} L[y]\right)-L^{-1}\left(\frac{p_{3}}{s^{2}+p_{1} s} L[f(y)]\right)
$$

where $F(x)$ represents the term arising from the source term and the prescribed initial conditions.

Now, we apply homotopy perturbation method [12],

$$
y(x)=\sum_{n=0}^{\infty} p^{n} y_{n}(x)
$$

where the term $y_{n}$ are to recursively calculated and the nonlinear term $f(y)$ can be decomposed as

$$
f(y)=\sum_{n=0}^{\infty} p^{n} H_{n}(y)
$$

for some He's polynomial $H_{n}$ (see [11] [19]) that are given by

$$
H_{n}\left(y_{0}, y_{1}, y_{2}, \cdots, y_{n}\right)=\frac{1}{n !} \frac{\partial^{n}}{\partial p^{n}}\left[f\left(\sum_{i=0}^{\infty} p^{i} y_{i}(x)\right)\right]_{P=0} \quad n=0,1,2,3, \cdots
$$

Substituting Equations (18) and (19) in (17), we get 


$$
\sum_{n=0}^{\infty} p^{n} y_{n}(x)=F(x)-p\left(L^{-1}\left\{\frac{p_{2}}{s^{2}-p_{1} s} L\left[\sum_{n=0}^{\infty} p^{n} y_{n}(x)\right]\right\}+L^{-1}\left\{\frac{p_{3}}{s^{2}+p_{1} s} L\left[\sum_{N=0}^{\infty} p^{n} H_{n}(y)\right]\right\}\right)
$$

which is the coupling of the Laplace transformation and the homotopy perturbation method using He's polynomials. Comparing the coefficient of like powers of $p$, the following approximations are obtained:

$$
\begin{aligned}
& p^{0}: y_{0}(x)=F(x), \\
& p^{1}: y_{1}(x)=-\left(L^{-1}\left\{\frac{p_{2}}{s^{2}+p_{1} s} L\left[y_{0}(x)\right]\right\}+L^{-1}\left\{\frac{p_{3}}{s^{2}+p_{1} s} L\left[H_{0}(y)\right]\right\}\right) \\
& p^{2}: y_{2}(x)=-\left(L^{-1}\left\{\frac{p_{2}}{s^{2}+p_{1} s} L\left[y_{1}(x)\right]\right\}+L^{-1}\left\{\frac{p_{3}}{s^{2}+p_{1} s} L\left[H_{1}(y)\right]\right\}\right) \\
& p^{3}: y_{3}(x)=-\left(L^{-1}\left\{\frac{p_{2}}{s^{2}+p_{1} s} L\left[y_{2}(x)\right]\right\}+L^{-1}\left\{\frac{p_{3}}{s^{2}+p_{1} s} L\left[H_{2}(y)\right]\right\}\right)
\end{aligned}
$$

\section{A Domain Decomposition Method}

A domain decomposition method [3] [4] define the unknown function $u(x)$ by an infinite series

$$
u(x)=\sum_{n=0}^{\infty} u_{n}(x)
$$

where the components $u_{n}(x)$, are usually determined recurrently. The nonlinear operator $F(u)$ can be decomposed into an infinite series of polynomials given by

$$
F(u)=\sum_{n=0}^{\infty} A_{n}
$$

where $A_{n}$ are the so-called Adomain polynomial of $u_{0}, u_{1} u_{2}, \cdots, u_{n}$ defined by

$$
A_{n}=\frac{1}{n !} \frac{\mathrm{d}^{n}}{\mathrm{~d} \lambda^{n}}\left[F\left(\lambda^{i} u_{i}\right)\right]_{\lambda=0}, n=0,1,2, \cdots
$$

or equivalently

$$
\begin{aligned}
& A_{0}=F\left(u_{0}\right), \\
& A_{1}=u_{1} F^{\prime}\left(u_{0}\right), \\
& A_{2}=u_{2} F^{\prime}\left(u_{0}\right)+\frac{1}{2} u_{1}^{2} F^{\prime \prime}\left(u_{0}\right), \\
& A_{3}=u_{3} F^{\prime}\left(u_{0}\right)+u_{1} u_{2} F^{\prime \prime}\left(u_{0}\right)+\frac{1}{3} u_{1}^{3} F^{\prime \prime \prime}\left(u_{0}\right), \\
& A_{4}=u_{4} F^{\prime}\left(u_{0}\right)+\left(u_{1} u_{3}+\frac{1}{2} u_{2}^{2}\right) F^{\prime \prime}\left(u_{0}\right)+\frac{1}{2} u_{1}^{2} u_{2} F^{\prime \prime \prime}\left(u_{0}\right)+\frac{1}{24} u_{1}^{4} F^{(\mathrm{iv})}\left(u_{0}\right) .
\end{aligned}
$$

It is now well known that these polynomials can be generated for all classes of nonlinear according to specific algorithms defined by (24). Recently, an alternative algorithm for constructing Adomain polynomials has been developed by Wazwaz [6].

This powerful technique handles both linear and nonlinear equations in unified manner without any need for the so-called Adomain polynomials. However, Adomin decomposition method provides the component of the exact solution, where these components should follow the summation given in (22), whereas ADM requires the evaluation of the Adomain polynomials that mostly require tedious algebraic work. 


\section{Applications}

\subsection{Example 1}

Consider the following nonlinear PDE [20]:

$$
\nabla^{2} u+\left(\frac{\partial u}{\partial y}\right)=2 y+x^{4}
$$

with the following conditions:

$$
\begin{array}{ll}
u(x, 0)=a x, & u(x, 1)=x(x+a), \\
u(0, y)=0, & u(1, y)=y+a
\end{array}
$$

\subsubsection{Using He-Laplace Method}

Equation (22) can be written as

$$
\frac{\partial^{2} u}{\partial x^{2}}+\frac{\partial^{2} u}{\partial y^{2}}+\left(\frac{\partial u}{\partial y}\right)^{2}=2 y+x^{4}
$$

By applying the Laplace transform to both sides of Equation (24) subject to the initial condition, we have

$$
L[u]=\frac{1}{s}\left(L[2 y]+L\left[x^{4}\right]\right)-\frac{1}{s^{2}} L\left(u_{x x}+u_{x x}^{2}\right)
$$

The inverse of the Laplace transform implies that

$$
u(x, y)=x^{2} y+\frac{x^{6}}{30}-p L^{-1}\left(\frac{1}{s^{2}} L\left[u_{y y}\right]+\frac{1}{s^{2}} L\left[u_{y}^{2}\right]\right)
$$

Now, we apply the homotopy perturbation method, we have

$$
\sum_{n=0}^{\infty} p^{n} u_{n}(x, y)=x^{2} y+\frac{x^{6}}{30}-p L^{-1}\left(\frac{1}{s^{2}}\left\{L\left[\sum_{n=0}^{\infty} p^{n} u_{n}\right]+\frac{1}{s^{2}} L\left[\sum_{n=0}^{\infty} p^{n} H_{n}(u)\right]\right\}\right)
$$

where $H_{n}(u)$ are He's polynomials. The first few com-ponents of He's polynomials are given by

$$
\begin{aligned}
& H_{0}(u)=y_{0 y}^{2}=x^{2} \\
& H_{1}(u)=2 y_{0 y} y_{1 y} \\
& H_{2}(u)=y_{1 y}^{2}+2 y_{0 y} y_{2 y}=0
\end{aligned}
$$

Comparing the coefficient of like powers of $p$, we have $p^{0}: u_{0}(x, y)=x^{2} y+\frac{x^{6}}{30}$, but we consider $u_{0}(x, y)=x^{2} y+\frac{x^{6}}{30}+\alpha x$

$$
\begin{aligned}
& p^{1}: u_{1}(x, y)=-L^{-1}\left[\frac{1}{s^{2}}\left\{L\left[y_{0 y y}\right]+L\left[H_{0}(y)\right]\right\}\right]=-\frac{x^{6}}{30} \\
& p^{2}: u_{2}(x, y)=-L^{-1}\left[\frac{1}{s^{2}}\left\{L\left[y_{1 y y}\right]+L\left[H_{1}(y)\right]\right\}\right]=0 \\
& p^{3}: u_{3}(x, y)=-L^{-1}\left[\frac{1}{s^{2}}\left\{L\left[y_{2 y y}\right]+L\left[H_{2}(y)\right]\right\}\right]=0
\end{aligned}
$$


So that the solution $u(x, y)$ is given by

$$
u(x, y)=u_{0}+u_{1}+u_{2}+u_{3}+\cdots=x^{2} y+\frac{x^{6}}{30}+a x-\frac{x^{6}}{30}+0+0+\cdots=x^{2} y+a x
$$

which is the exact solution of the problem.

\subsubsection{Adomain Decomposition Method}

We first rewrite Equation (26) in an operator $L$ is

$$
\begin{gathered}
\frac{\partial^{2} u}{\partial x^{2}}+\frac{\partial^{2} u}{\partial y^{2}}+\left(\frac{\partial u}{\partial y}\right)^{2}=2 y+x^{4} \\
L_{x x} u+L_{y y} u+\left(L_{y} u\right)^{2}=2 y+x^{4} \\
L_{x x} u=2 y+x^{4}-L_{y y} u-\left(L_{y} u\right)^{2} \\
u(x, 0)=a x, u(x, 1)=x(x+a) \\
u(0, y)=0, u(1, y)=y+a
\end{gathered}
$$

where the differential operators $L_{x x}, L_{y y} \& L_{y}$ are

$$
L_{x x}(.)=\frac{\partial^{2}}{\partial x^{2}}(.), L_{y y}(.)=\frac{\partial^{2}}{\partial y^{2}}(.) \& L_{y}(.)=\frac{\partial}{\partial y}(.)
$$

The inverse $L_{x x}^{-1}$ are assumed as an integral operator given by

$$
L_{x x}^{-1}(.)=\int_{0}^{x} \int_{0}^{x}(.) \mathrm{d} x \mathrm{~d} x
$$

Appling the inverse operator $L_{x x}^{-1}$ on both sides of (35) and using initial condition we find

$$
u(x, y)=y x^{2}+\frac{1}{30} x^{6}+a x-L_{x x}^{-1}\left(L_{y y} u+\left(L_{y} u\right)^{2}\right)
$$

Substituting (22) into the function Equation (38) gives

$$
\sum_{n=0}^{\infty} u_{n}(x, y)=y x^{2}+\frac{1}{30} x^{6}+a x-L_{x x}^{-1}\left(L_{y y} \sum_{n=0}^{\infty} u_{n}(x, y)+\left(L_{y} \sum_{n=0}^{\infty} u_{n}(x, y)\right)^{2}\right)
$$

This can be rewrite at the form

$$
\begin{aligned}
& u_{0}+u_{1}+u_{2}+\cdots \\
& =x^{2} y+\frac{1}{30} x^{6}+a x-L_{x x}^{-1}\left(L_{y y}\left(u_{0}+u_{1}+u_{2}+\cdots\right)+\left(L_{y}\left(u_{0}+u_{1}+u_{2}+\cdots\right)\right)^{2}\right)
\end{aligned}
$$

In view of (39), the following recursive relation

$$
\begin{aligned}
& u_{0}=x^{2} y+\frac{1}{30} x^{6}+a x \\
& u_{k+1}(x, y)=-L_{x x}^{-1}\left(L_{y y} u_{k}(x, y)+\left(L_{y} u_{k}(x, y)\right)^{2}\right), k \geq 0
\end{aligned}
$$

follows immediately. Consequently, we obtain 


$$
\begin{aligned}
& u_{0}=x^{2} y+\frac{1}{30} x^{6}+a x \\
& u_{1}=-L_{x x}{ }^{-1}\left(L_{y y} u_{0}(x, y)+\left(L_{y} u_{0}(x, y)\right)^{2}\right) \\
& u_{2}=-L_{x x}{ }^{-1}\left(L_{y y} u_{2}(x, y)+\left(L_{y} u_{2}(x, y)\right)^{2}\right)
\end{aligned}
$$

According to Adomain [19], and approximate solution can be obtained [12].

$$
u(x, y)=x(x y+a)+\left(\frac{1}{2}\right)\left\{y(y-1)\left(\frac{x^{4}}{2}+\frac{y+1}{3}\right)+\frac{x}{30}\left(x^{5}-1\right)\right\}
$$

the exact solution is given by $u(x, y)=x(x y+a)$.

\subsection{Example 2}

Consider the following non-homogeneous nonlinear PDE [20]:

with the following condition:

$$
\frac{\partial u}{\partial t}=x^{2}-\left(\frac{1}{4}\right)\left(\frac{\partial u}{\partial x}\right)^{2}
$$

$$
u(x, 0)=0
$$

\subsubsection{Using He-Laplace Method}

By applying the Laplace transform method subject to the initial condition, we have

$$
y(x, s)=\frac{L\left[x^{2}\right]}{s}-\frac{1}{4 s} L\left[u_{x}^{2}\right]
$$

The inverse of the Laplace transform implies that

$$
u(x, t)=x^{2} t-L^{-1}\left[\frac{1}{4 s} L\left[u_{x}^{2}\right]\right]
$$

Now, we apply the homotopy perturbation method, we have

$$
\sum_{n=0}^{\infty} p^{n} u_{n}(x, t)=x^{2} t-p\left(L^{-1}\left[\frac{1}{4 s}\left\{L\left[\sum_{n=0}^{\infty} p^{n} u_{n}(u)\right]\right\}\right]\right)
$$

where $H_{n}(u)$ are He's polynomials. The first few components of He's polynomials are given by

$$
\begin{aligned}
& H_{0}(u)=u_{0 x}^{2}=4 x^{2} t^{2} \\
& H_{1}(u)=2 u_{0 x} u_{1 x}=-\frac{8 x^{2} t^{4}}{3} \\
& H_{2}(u)=u_{1 x}^{2}+2 u_{0 x} u_{2 x}=\frac{2 x^{2} t^{3}}{9}+\frac{16 x^{2} t^{6}}{15}
\end{aligned}
$$

Comparing the coefficient of like powers of $p$ we have

$$
\begin{aligned}
& p^{0}: u_{0}(x, t)=x^{2} t \\
& p^{1}: u_{1}(x, t)=-L^{-1}\left[\frac{1}{4 s}\left\{L\left[H_{0}(u)\right]\right\}\right]=\frac{x^{2} t^{3}}{3} \\
& p^{2}: u_{2}(x, t)=-L^{-1}\left[\frac{1}{4 s}\left\{L\left[H_{1}(u)\right]\right\}\right]=\frac{2 x^{2} t^{5}}{15}
\end{aligned}
$$


Proceeding in a similar manner, we have

$$
p^{3}: u_{3}(x, t)=-\frac{17 x^{2} t^{7}}{315}
$$

So that the solution $u(x, t)$ is given by

$$
u(x, t)=u_{0}+u_{1}+u_{2}+u_{3}+\cdots=x^{2} t-\frac{x^{2} t^{3}}{3}+\frac{2 x^{2} t^{5}}{15}-\frac{17 x^{2} t^{7}}{315}+\cdots
$$

\subsubsection{Adomain Decomposition Method}

We first rewrite Equation (44) in an operator $L$ is

$$
\begin{gathered}
L_{t} u=x^{2}-\frac{1}{4}\left(L_{x} u\right)^{2} \\
u(x, 0)=0
\end{gathered}
$$

where the differential operators are define as;

$$
L_{t}(\cdot)=\frac{\partial}{\partial t}(\cdot), \quad L_{x}(\cdot)=\frac{\partial}{\partial x}(\cdot)
$$

And the inverse operator $L_{t}^{-1}$, provided that it exists, is defined as:

$$
L_{t}^{-1}(\cdot)=\int_{0}^{t}(\cdot) \mathrm{d} t
$$

Appling the inverse operator on both the sides of (52) and using the initial condition, yields:

$$
\begin{gathered}
L_{t}^{-1}\left(L_{t} u\right)=L_{t}^{-1}\left(x^{2}\right)-\frac{1}{4} L_{t}^{-1}\left(L_{x} u\right)^{2} \\
u(x, t)=x^{2} t-\frac{1}{4} L_{t}^{-1}\left(L_{x} u\right)^{2}
\end{gathered}
$$

Now, we decompose the unknown function $u(x, t)$ as a sum of components defined by the series (22):

$$
u(x, t)=\sum_{n=0}^{\infty} u_{n}(x, t)
$$

where $u_{0}$ is identified as $u(x ; 0)$. The components $u_{n}(x, t)$ are obtained by the recursive formula:

$$
\sum_{n=0}^{\infty} u_{n}(x, t)=x^{2} t-\frac{1}{4} L_{t}^{-1}\left(L_{x}\left(\sum_{n=0}^{\infty} u_{n}(x, t)\right)\right)^{2}
$$

Or

$$
\begin{gathered}
u_{0}(x, t)=x^{2} t \\
u_{k+1}(x, t)=-\frac{1}{4} L_{t}^{-1}\left(L_{x}\left(u_{k}(x, t)\right)\right)^{2}, k \geq 0
\end{gathered}
$$

We note that the recursive relationship is constructed on the basis that the component $u_{0}(x, t)$ is defined by all terms that arise from the initial condition and from integrating the source term. The remaining components $u_{k}(x, t)$, can be completely determined recursively.

Accordingly, considering the first few terms, Equations (14) and (15) give: 


$$
\begin{aligned}
u_{0} & =x^{2} t \\
u_{1} & =-\frac{1}{4} L_{t}^{-1}\left(L_{x}\left(u_{0}(x, t)\right)\right)^{2}=-\frac{1}{4} L_{t}^{-1}\left(L_{x}\left(x^{2} t\right)\right)=-\frac{x^{2} t^{2}}{3} \\
u_{2} & =-\frac{1}{4} L_{t}^{-1}\left(L_{x}\left(u_{2}(x, t)\right)\right)^{2}=-\frac{x^{2} t^{7}}{63} \\
& :
\end{aligned}
$$

Finally, using (55) we obtain the solution in series form:

$$
u(x, t)=u_{0}+u_{1}+u_{2}+u_{3}+\cdots
$$

That is:

$$
u(x, t)=x^{2} t-\frac{x^{2} t^{3}}{3}-\frac{x^{2} t^{7}}{63}+\cdots
$$

\subsection{Example 3}

Consider the following first order nonlinear differential equation [19]

$$
y^{\prime}+y^{2}=0, \quad y \geq 0
$$

With the following condition:

$$
y(0)=1
$$

\subsubsection{Using He-Laplace Method}

By applying the aforesaid method subject to the initial condition, we have

$$
L[y(x)]=\frac{1}{S}-\frac{1}{S} L\left[y^{2}\right]
$$

The inverse of Laplace transform implies that

$$
y(x)=1-L^{-1}\left[\frac{1}{s} L\left[y^{2}\right]\right]
$$

Now we apply the homotopy perturbation method, we have

$$
\sum_{n=0}^{\infty} p^{n} y_{n}(x)=1-p\left(L^{-1}\left[\frac{1}{s} L\left[\sum_{n=0}^{\infty} p^{n} H_{n}(y)\right]\right]\right)
$$

where $H_{n}(y)$ are He's polynomials. The first few components of He's polynomials are given by

$$
\begin{aligned}
& H_{0}(y)=y_{0}^{2}=1 \\
& H_{1}(y)=2 y_{0} y_{1}=-2 x \\
& H_{2}(y)=y_{1}^{2}+2 y_{0} y_{2}=3 x^{2}
\end{aligned}
$$

Comparing the coefficient of like powers of $p$, we have

$$
\begin{aligned}
& p^{0}: y_{0}(x)=1 \\
& p^{1}: y_{1}(x)=-L^{-1}\left[\frac{1}{s}\left\{L\left[H_{0}(y)\right]\right\}\right]=-x \\
& p^{2}: y_{2}(x)=-L^{-1}\left[\frac{1}{s}\left\{L\left[H_{1}(y)\right]\right\}\right]=x^{2} \\
& p^{3}: y_{3}(x)=-L^{-1}\left[\frac{1}{s}\left\{L\left[H_{2}(y)\right]\right\}\right]=-x^{3}
\end{aligned}
$$


So that the solution $y(x)$ is given by

$$
\begin{aligned}
& y(x)=y_{0}+y_{1}+y_{2}+y_{3}+\cdots \\
& y(x)=1-x+x^{2}-x^{3}+\cdots
\end{aligned}
$$

which is converging to $\left(\frac{1}{1+x}\right)$ i.e. exact solution.

\subsubsection{Adomain Decomposition Method}

We first rewrite Equation (61) in an operator $L$ is

$$
\begin{aligned}
& L y=-y^{2} \\
& y(0)=1
\end{aligned}
$$

where the differential operators are define as;

$$
L(\cdot)=\frac{\partial}{\partial t}(\cdot)
$$

And the inverse operator $L^{-1}$ provided that it exists, is defined as

$$
L^{-1}(\cdot)=\int_{0}^{t}(\cdot) \mathrm{d} t
$$

Appling the inverse operator on both the sides of (69) and using the initial condition yields:

$$
\begin{gathered}
L^{-1}(L y)=L^{-1}\left(-y^{2}\right) \\
y(x)=1-L^{-1}\left(y^{2}\right)
\end{gathered}
$$

Now, we decompose the unknown function $y(t)$ as a sum of components defined by the series (22):

$$
u(x)=\sum_{n=0}^{\infty} u_{n}(x)
$$

where $y_{0}$ is identified as $y(0)$. The components $y_{k}(t)$ are obtained by the recursive formula:

$$
\sum_{n=0}^{\infty} y_{n}(t)=1-L^{-1}\left(\left(\sum_{n=0}^{\infty} y_{n}^{2}\right)\right)
$$

Or

$$
\begin{gathered}
y_{0}=1 \\
y_{k+1}(t)=-L^{-1}\left(y_{k}^{2}\right)
\end{gathered}
$$

We note that the recursive relationship is constructed on the basis that the component $y_{0}(t)$ is defined by all terms that arise from the initial condition and from integrating the source term. The remaining components $y_{k}(t)$, can be completely determined recursively.

Accordingly, considering the first few terms, Equations (72) and (73) give:

$$
\begin{aligned}
& y_{0}=1 \\
& y_{1}(t)=-L^{-1}\left(y_{0}^{2}\right)=-L^{-1}(1)=-t \\
& y_{2}(t)=-L^{-1}\left(y_{1}^{2}\right)=-L^{-1}\left(t^{2}\right)=-\frac{t^{3}}{3} \\
& y_{3}(t)=-L^{-1}\left(y_{2}^{2}\right)=-L^{-1}\left(\frac{t^{6}}{9}\right)=-\frac{t^{7}}{63}
\end{aligned}
$$


Finally, using (55) we obtain the solution in series form:

$$
y(x)=y_{0}+y_{1}+y_{2}+y_{3}+\cdots
$$

That is:

$$
y(x)=1-t-\frac{t^{3}}{3}-\frac{t^{7}}{63}
$$

\section{Discussions}

The main goal of this work is to conduct a comparative study between Adomain decomposition method and the He-Laplace method. The two methods are powerful and efficient methods that both give approximations of higher accuracy and closed form solutions if existing.

An important conclusion can be made here. Adomain decomposition method for solving nonlinear ordinary and partial differential equations, the same problems are solved by He-Laplace method. Adomain decomposition method provides the components of exact solution, where these components should follow the summation given in (22). However, He-Laplace is an elegant combination of the Laplace transformation, the homotopy perturbation method and He's polynomials. Moreover, the ADM requires the evaluation of the Adomain polynomial that mostly require tedious algebraic calculations. The ADM provides the solution in successive components that will be added to get the series solution.

\section{References}

[1] Adomian, G. (1988) A Review of the Decomposition Method in Applied Mathematics. Journal of Mathematical Analysis and Applications, 135, 501-544. http://dx.doi.org/10.1016/0022-247X(88)90170-9

[2] Adomian, G. (1994) Solving Frontier Problems of Physics: The Decomposition Method. Kluwer Academic Publishers, Boston.

[3] Wazwaz, A.M. (1997) Necessary Conditions for the Appearance of Noise Terms in Decomposition Solution Series. Applied Mathematics and Computation, 81, 265-274. http://dx.doi.org/10.1016/S0096-3003(95)00327-4

[4] Wazwaz, A.M. (1997) A First Course in Integral Equations. World Scientific, Singapore. http://dx.doi.org/10.1142/3444

[5] Wazwaz, A.M. (1999) Analytical Approximations and Padé's Approximants for Volterra’s Population Model. Applied Mathematics and Computation, 100, 13-25. http://dx.doi.org/10.1016/S0096-3003(98)00018-6

[6] Wazwaz, A.M. (2000) A New Technique for Calculating Adomian Polynomials for Nonlinear Polynomials. Applied Mathematics and Computation, 111, 33-51. http://dx.doi.org/10.1016/S0096-3003(99)00063-6

[7] Wazwaz, A.M. (2000) A New Algorithm for Calculating Adomian Polynomials for Nonlinear Operators. Applied Mathematics and Computation, 111, 53-69.

[8] Wazwaz, A.M. (2000) The Decomposition Method for Solving the Diffusion Equation Subject to the Classification of Mass, Internat. Applied Mathematics and Computation, 3, 25-34.

[9] Wazwaz, A.M. (2002) Partial Differential Equations: Methods and Applications. Balkema Publishers, The Netherlands.

[10] Wazwaz, A.M. (2002) A New Method for Solving singular Initial Value Problems in the Second Order Differential Equations. Applied Mathematics and Computation, 128, 47-57. http://dx.doi.org/10.1016/S0096-3003(01)00021-2

[11] Ghorbani, A. (2009) Beyond Adomian’s Polynomials: He’s Polynomials. Chaos, Solitons Fractals, 39, $1486-1492$. http://dx.doi.org/10.1016/j.chaos.2007.06.034

[12] Lyapunov, A.M. (1992) The General Problem of the Stability of Motion. Taylor \& Francis, London.

[13] Saberi-Nadjafi, J. and Ghorbani, A. (2009) He’s Homotopy Per-turbation Method: An Effective Tool for Solving Nonlinear Integral and Integro-Differential Equations. Computers and Mathematics with Applications, 58, 1345-1351.

[14] Sweilam, N.H. and Khadar, M.M. (2009) Exact Solution of Some Coupled Nonlinear Partial Differential Equations Using the Homotopy Perturbation Method. Computers and Mathematics with Applications, 58, 2134-2141. http://dx.doi.org/10.1016/j.camwa.2009.03.059

[15] Hirota, R. (1971) Exact Solutions of the Korteweg-de Vries Equation for Multiple Collisions of Solitons. Physics Review Letters, 27, 1192-1194. 
[16] Wazwaz, A.M. (2010) On Multiple Soliton Solution for Coupled KdV-mkdV Equation. Nonlinear Science Letter A, 1, 289-296.

[17] Wu, G.C. and He, J.H. (2010) Fractional Calculus of Variations in Fractal Space Time. Nonlinear Science Letter A, 1, 281-287.

[18] He, J.H. (2003) A Simple Perturbation Approach to Blasius Equation. Applied Mathematics and Computation, 140, 217-222. http://dx.doi.org/10.1016/S0096-3003(02)00189-3

[19] Liu, G.L. (1995) Weighted Residual Decomposition Method in Nonlinear Applied Mathematics. Proceedings of 6th Congress of Modern Mathematics and Mechanics, Suzhou, 1995, 643-648.

[20] He, J.H. (1997) A New Approach to Nonlinear Partial Differential Equations. Communications in Nonlinear and Numerical Simulation, 2, 230-235. http://dx.doi.org/10.1016/S1007-5704(97)90007-1 
Scientific Research Publishing (SCIRP) is one of the largest Open Access journal publishers. It is currently publishing more than 200 open access, online, peer-reviewed journals covering a wide range of academic disciplines. SCIRP serves the worldwide academic communities and contributes to the progress and application of science with its publication.

Other selected journals from SCIRP are listed as below. Submit your manuscript to us via either submit@scirp.org or Online Submission Portal.
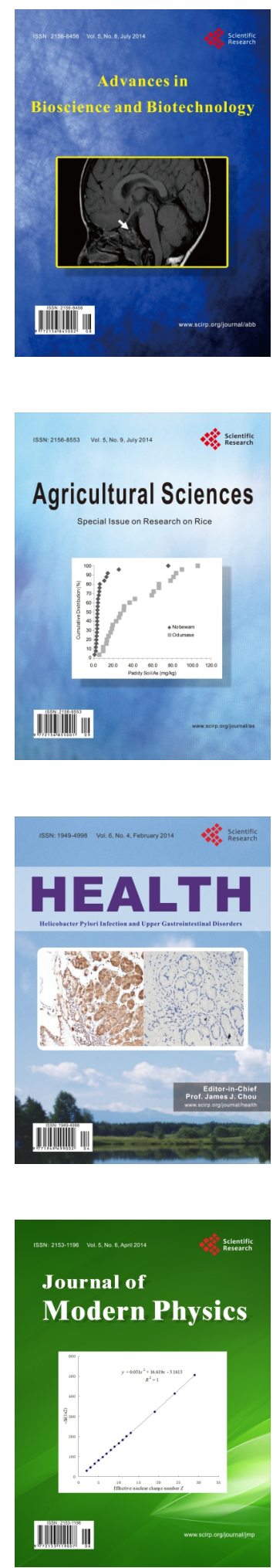
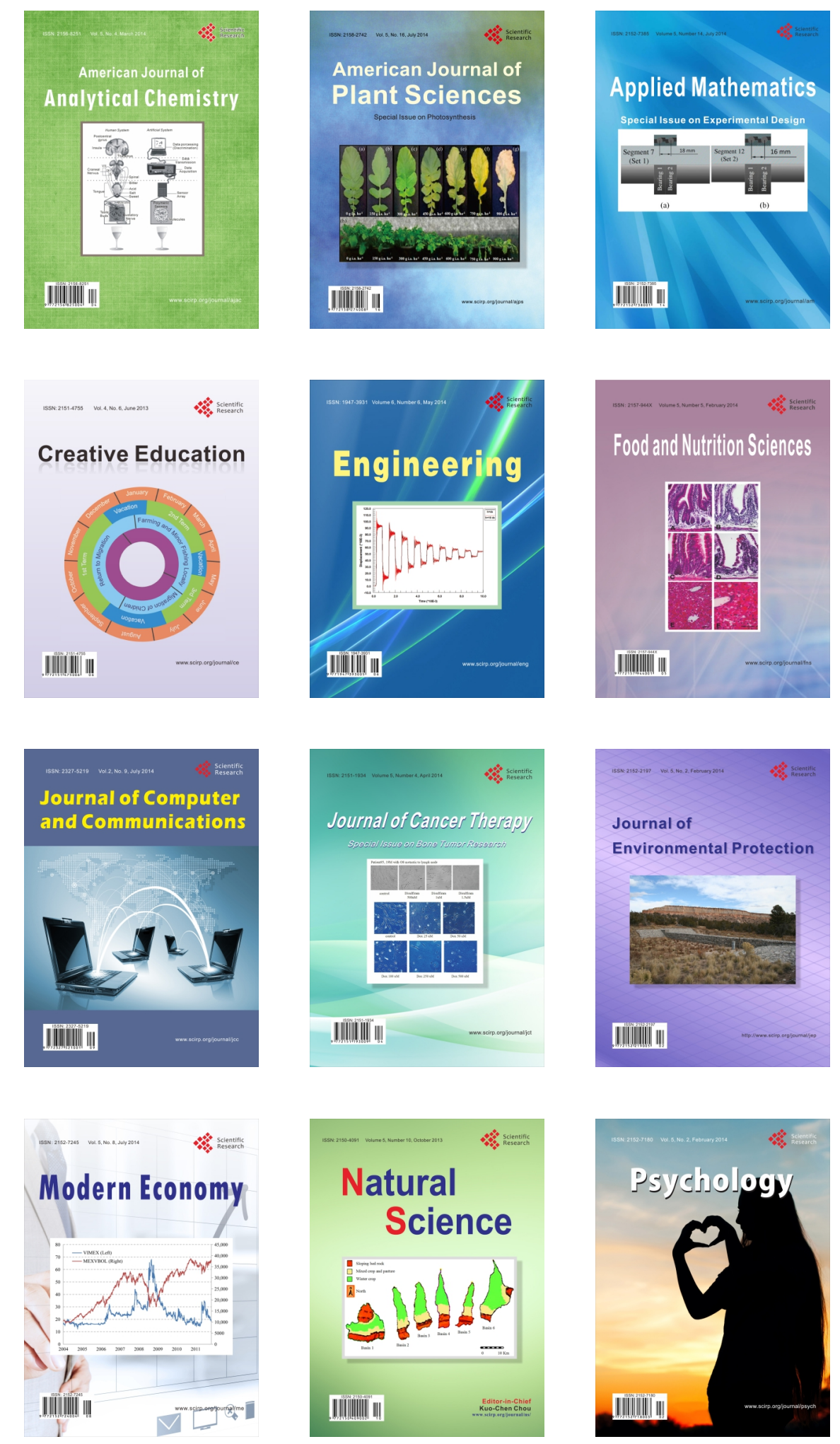